\title{
MIAWARA-C, a new ground based water vapor radiometer for measurement campaigns
}

\author{
C. Straub, A. Murk, and N. Kämpfer \\ Institute of Applied Physics, University of Bern, Switzerland \\ Received: 18 May 2010 - Published in Atmos. Meas. Tech. Discuss.: 28 May 2010 \\ Revised: 12 August 2010 - Accepted: 8 September 2010 - Published: 23 September 2010
}

\begin{abstract}
In this paper a new $22 \mathrm{GHz}$ water vapor spectroradiometer which has been specifically designed for profile measurement campaigns of the middle atmosphere is presented. The instrument is of a compact design and has a simple set up procedure. It can be operated as a standalone instrument as it maintains its own weather station and a calibration scheme that does not rely on other instruments or the use of liquid nitrogen. The optical system of MIAWARA-C combines a choked gaussian horn antenna with a parabolic mirror which reduces the size of the instrument in comparison with currently existing radiometers. For the data acquisition a correlation receiver is used together with a digital cross correlating spectrometer. The complete backend section, including the computer, is located in the same housing as the instrument. The receiver section is temperature stabilized to minimize gain fluctuations. Calibration of the instrument is achieved through a balancing scheme with the sky used as the cold load and the tropospheric properties are determined by performing regular tipping curves. Since MIAWARA-C is used in measurement campaigns it is important to be able to determine the elevation pointing in a simple manner as this is a crucial parameter in the calibration process. Here we present two different methods; scanning the sky and the Sun. Finally, we report on the first spectra and retrieved water vapor profiles acquired during the Lapbiat campaign at the Finnish Meteorological Institute Arctic Research Centre in Sodankylä, Finland. The performance of MIAWARA-C is validated here by comparison of the presented profiles against the equivalent profiles from the Microwave Limb Sounder on the EOS/Aura satellite.
\end{abstract}

Correspondence to: C. Straub (corinne.straub@iap.unibe.ch)

\section{Introduction}

Water vapor is a key element in the Earth's radiative budget as it is the most important greenhouse gas in the upper troposphere and contributes to the radiative cooling of the stratosphere by infrared emission. Alongside this it is also involved in several chemical processes, one of which is ozone depletion, where it acts as the main source of the reactive $\mathrm{OH}$ radical. In the stratosphere water vapor has a long chemical lifetime with respect to the time scale of dynamical processes and can therefore be used as a tracer to investigate phenomena such as stratosphere-troposphere exchange, meridional transport and polar vortex containment.

Approximately half of the water vapor in the middle atmosphere enters it by vertical transport through the tropical tropopause where solar irradiation is at its greatest, creating a strong upward stream of air called the "tropical pipe", which reaches from the troposphere up to the stratosphere (Plumb, 1996). The other main source of middle atmospheric water vapor is the oxidation of methane leading to an increase in the volume mixing ratio with increasing altitude in the stratosphere. Photo-dissociation due to the absorption of solar UVradiation is the only relevant sink of water vapor in the middle atmosphere, leading to a decrease in the volume mixing ratio with increasing altitude throughout the mesosphere.

Water vapor in the upper stratosphere and mesosphere is mainly observed by passive remote sensing instruments, either space borne or ground based. While satellite instruments, such as MLS on EOS/Aura (Waters et al., 2006), MIPAS on ENVISAT (Milz et al., 2005) and ODIN (Murtagh et al., 2002) provide the vertical as well as the horizontal distribution of water vapor, ground based instruments provide vertical profiles in a single location. Ground based instruments are characterized by long operational lifetimes while the lifetime for satellites is typically limited to less

Published by Copernicus Publications on behalf of the European Geosciences Union. 
than 10 years. Therefore measurements from the ground are important for long term monitoring of water vapor and the merging of consecutive satellite missions. Thus, long term global datasets can be generated, which are crucial for climate research.

For middle atmospheric water vapor profiling from the ground the rotational transition at $22 \mathrm{GHz}$ is typically used since at this frequency the tropospheric opacity is low and therefore this line can be observed even from sea level. There are few ground based spectro-radiometers at $22 \mathrm{GHz}$ operating on a regular basis, e.g. the instruments Andoya (Norway), Seoul (South Korea) (de Wachter et al., 2010) in addition to the NDACC (Network for the Detection for Atmospheric Composition Change) instruments in Onsala (Sweden) (Forkman et al., 2003), Bern (Switzerland) (Deuber et al., 2004), Table Mountain (California, USA), Mauna Loa (Hawaii, USA) and Lauder (New Zealand) (Thacker et al., 1995), (Nedoluha et al., 1995) and (Nedoluha et al., 2007). There have also been several new developments in recent years, e.g. the instruments on the Zugspitze (Germany), Kuehlungsborn (Germany), Karlsruhe (Germany) and the Mobile Microwave Radiometer operated by the University of Toulouse (France) (Motte et al., 2008).

The instrument we present here is the third $22 \mathrm{GHz}$ microwave radiometer for middle atmospheric water vapor built by the Institute of Applied Physics, University of Bern. The first was the Middle Atmospheric WAter vapor RAdiometer (MIAWARA) (Deuber et al., 2004) which started routine operation near Bern in 2002 and is part of NDACC since 2005. The second instrument, the Stratospheric WAter Vapor RAdiometer (SWARA) (de Wachter et al., 2010), is a joint project of the University of Bern and the Sookmyung Women's University of Seoul, South Korea and began operation in Seoul in 2006. It was designed as a copy of MIAWARA.

The motivation behind the construction of the new MIAWARA-C instrument presented here was to have a microwave instrument that could be easily transported and would therefore be well suited for use in measurement campaigns. A significant advantage to this design is that it can act as a traveling standard for intercomparisons between the other instruments currently in routine operation, and it can also participate in measurement campaigns as an independent ground based instrument covering an altitude range complementary to the altitudes of other profiling techniques like radiosondes, LIDAR and FTIR. MIAWARA-C, shown in Fig. 1, is a standalone instrument that requires only electrical power and an internet connection since the backend and the computer are included in the housing. The calibration concept is independent of any information from other instruments or liquid nitrogen and the rainhood closes automatically whenever there is precipitation or strong winds to prevent the instrument from damage. The temperature and ground pressure data needed for the calibration comes from a weather station (WTX520 from Vaisala) which is attached to

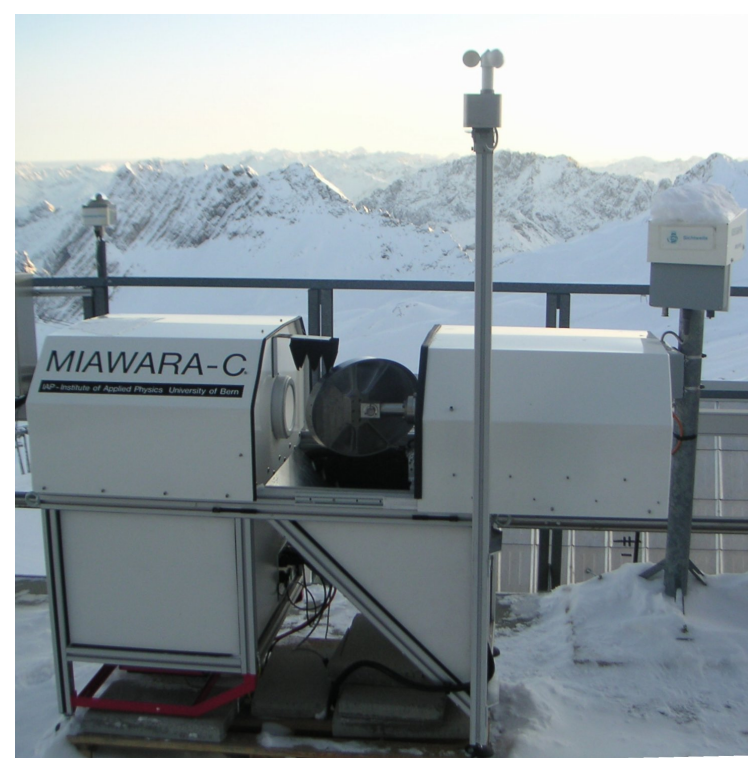

Fig. 1. MIAWARA-C during the ARIS-Campaign on the Zugspitze.

the instrument. In the second section of this paper we present the instrumental set-up of MIAWARA-C, focusing on the optical system and the correlation receiver. In the third section we present the calibration scheme used for MIAWARA-C, which is a hybrid between correlation receiver and external balancing. In the fourth section we describe two different methods for the validation of the instrumental pointing; scanning of the sky and the Sun. The fifth section is dedicated to a series of $\mathrm{H}_{2} \mathrm{O}$ spectra and profiles acquired with MIAWARA-C. We also provide comparisons of the results with those from Aura/MLS.

\section{Measurement principle and instrumental set up}

MIAWARA-C measures the spectral intensity of the pressure broadened water vapor emission line at $22.235 \mathrm{GHz}$. The shape of this line can be related to the vertical distribution of water vapor in the atmosphere because the pressure decreases exponentially with altitude. For MIAWARA-C, a retrieval algorithm based on the optimal estimation technique described in Rodgers (2000), is used to retrieve water vapor profiles from the measured spectra. In the middle atmosphere retrievals are possible at altitudes between approximately 30 and $80 \mathrm{~km}$ with the present setup. The upper limit is given by the frequency resolution of the spectrometer and the Doppler broadening effect while the lower limit is given by the bandwidth of the spectrometer and the distortion of the spectrum by instrumental artifacts, known as the baseline.

In microwave radiometry the intensity of radiation is usually expressed in terms of brightness temperature $T_{\mathrm{b}}$ according to the Rayleigh-Jeans approximation of Planck's law. The brightness temperature of the atmosphere along a certain 


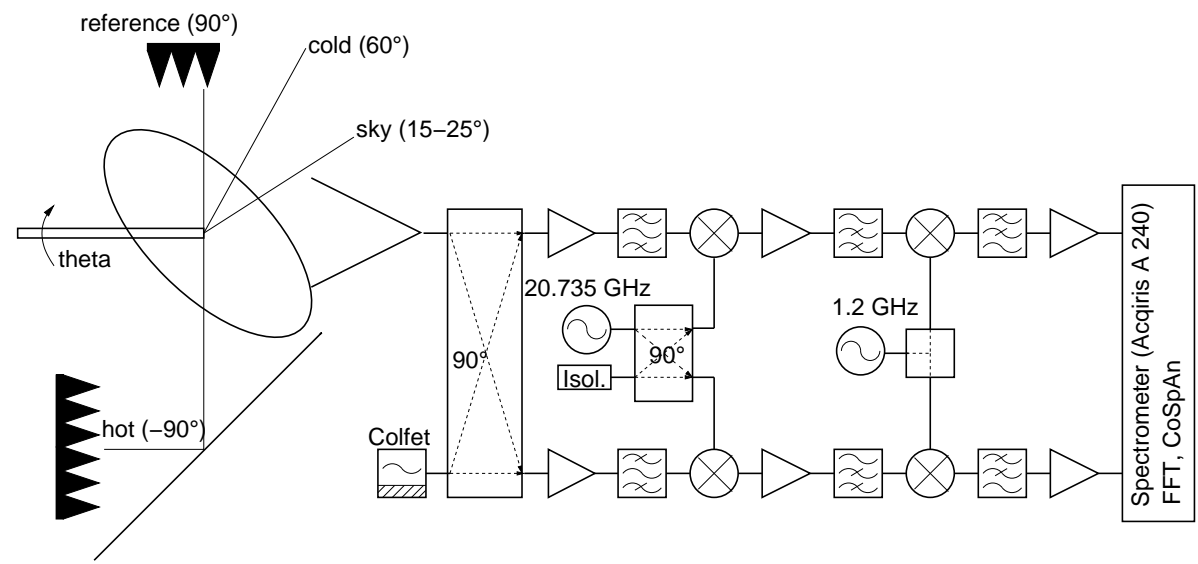

Fig. 2. Block diagram of the correlation receiver of MIAWARA-C.

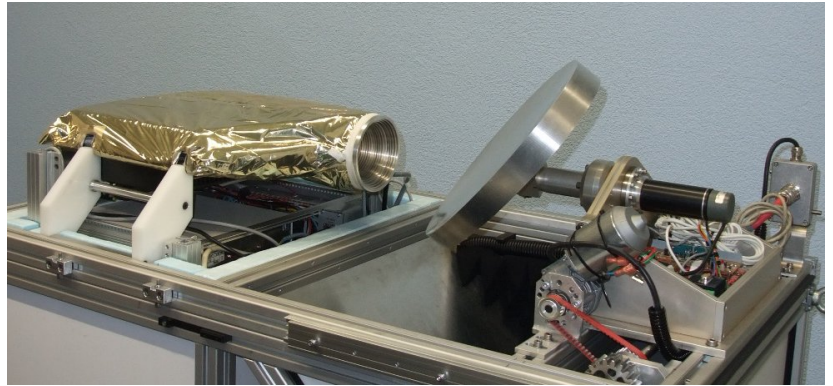

Fig. 3. Optical system of MIAWARA-C.

line of sight observed from the ground is a superposition of emission and absorption of radiation at different altitudes. It can be described by the radiative transfer equation (RTE)

$T_{\mathrm{b}}(\nu, 0)=T_{0} e^{-\tau\left(\nu, s_{0}\right)}+\int_{0}^{s_{0}} T(s) e^{-\tau(\nu, s)} \alpha(\nu, s) d s$

where $T_{\mathrm{b}}(v, 0)$ is the brightness temperature measured at frequency $v$ from an observation position 0 on the earth surface, $s_{0}$ the position of the upper boundary of the atmosphere, $T_{0}$ the microwave background temperature, $T(s)$ the physical temperature, and $\alpha(\nu, s)$ the frequency dependent absorption coefficient along the integration path. The opacity or optical depth $\tau$ is defined as the integral of $\alpha$ along the line of sight:

$\tau(v, s)=\int_{0}^{s} \alpha\left(v, s^{\prime}\right) d s^{\prime}$

An overview of the instrumental set-up and specifications of MIAWARA-C are given in Figs. 1, 2 and 3 and Table 1. As with most radiometers MIAWARA-C uses an optical system consisting of a horn antenna and a slewable mirror to direct the radiation from the atmosphere into a heterodyne receiver which amplifies and transforms the signal so it can be detected with a spectrometer. The optical and receiving system of MIAWARA-C are described in detail in the following sections.
Table 1. Key parameters and specifications of MIAWARA-C

\begin{tabular}{|c|c|}
\hline Optical system & $\begin{array}{l}\text { choked gaussian horn combined } \\
\text { with parabolic mirror, HPBW } 5.0^{\circ}\end{array}$ \\
\hline Receiver type & $\begin{array}{l}\text { correlation receiver with digital } \\
\text { cross correlation FFT spectrometer }\end{array}$ \\
\hline Receiver operation mode & Single side band \\
\hline RF-frequency range & $22.012-22.411 \mathrm{GHz}$ \\
\hline Frequency resolution & $30.5 \mathrm{kHz}$ \\
\hline Calibration & Balancing \\
\hline Absolute calibration & hot, cold \\
\hline
\end{tabular}

\subsection{Optical system}

The optical system of MIAWARA-C, shown in Fig. 3, consists of a compact optimized horn antenna and an off-axis parabolic mirror (Straub et al., 2007). Both the mirror and the horn antenna are mounted on the same profile frame which guarantees precise alignment, even during transportation of the instrument.

For middle atmospheric water vapor measurements a narrow antenna beam is needed, as the intensity of the intercepted radiation is dependent on the path length through the atmosphere, which is itself determined by the elevation of the antenna beam. The wider the antenna beam, the larger the elevation range that contributes to the received signal. The antenna beam of MIAWARA-C has a Half Power Beam Width (HPBW) of $5.0^{\circ}$, corresponding to a directivity of $31.7 \mathrm{~dB}$. To keep the antenna as small as possible a horn with a relatively low directivity of $23.8 \mathrm{~dB}$ was chosen and combined with a focusing parabolic mirror to achieve the required directivity of $31.7 \mathrm{~dB}$.

The horn antenna used for MIAWARA-C is a choked Gaussian horn (Teniente et al., 2002), whose geometry was optimized to achieve an antenna pattern of high rotational symmetry with sidelobes below $-40 \mathrm{~dB}$. During calibration the mirror is slewed, making the high rotational symmetry of 


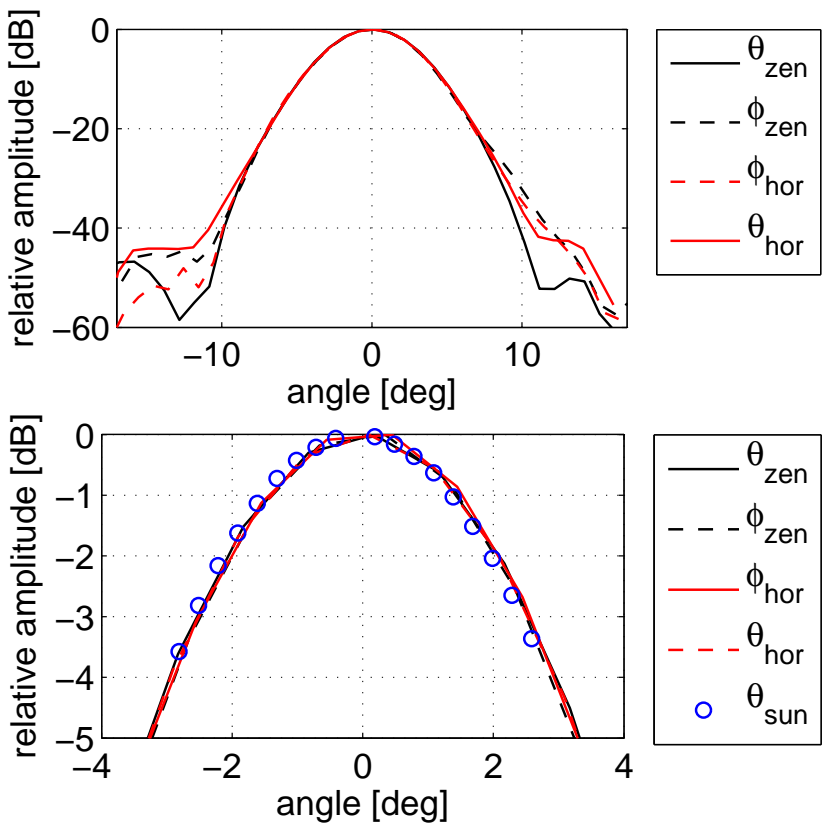

Fig. 4. Farfield at $22.235 \mathrm{GHz}$ of the optical system of MIAWARA-C measured with two different methods. Laboratory measurements deliver the farfield pattern in scanning direction $\theta$ and the direction $\phi$ orthogonal to the scanning plane for the zenith and the horizon. The measurement using the Sun as radiation source results in a pattern in scanning direction at the elevation angle of the Sun.

the horn antenna beam important. Were the beam in any way asymmetrical the calibration process would lead to a mismatch between the horn and the mirror and thus would introduce systematic errors in beam coupling. The low side lobe level is crucial so as to maintain the required high level of beam efficiency since the signal received from the stratosphere has a line amplitude of only approximately $0.2 \mathrm{~K}$. With higher sidelobe levels the radiometer would intercept power from directions other than from the atmosphere, leading to systematic errors in the antenna temperature.

To keep spillover losses small the parabolic mirror needs to be as large as possible. However, as MIAWARA-C is a campaign instrument that is required to travel, the mirror dimensions must also be kept reasonably small. Therefore, the size of the mirror is a trade off between those two requirements. The geometry of the mirror was optimized using physical optics simulations to maintain a constant edge taper below $-35 \mathrm{~dB}$. This resulted in an elliptical rim with major and minor axes of $39 \mathrm{~cm}$ and $27.8 \mathrm{~cm}$ respectively. A $90^{\circ}$ off axis geometry was chosen which provides the measurement geometry easiest for data processing.

Using the choked Gaussian antenna instead of a conventional conical corrugated horn antenna ensured a greater than threefold reduction in spillover loss for the optimized mirror from $0.21 \%$ to $0.06 \%$ of the received power. Another advan- tage of this choice of horn is its relatively short length (approximately $15 \mathrm{~cm}$ ). This is half the length of a conical corrugated horn having the same directivity. The choked Gaussian horn thus ensures a reduced mirror diameter and horn length, thus minimizing the dimensions of the overall instrument.

The plots in Fig. 4 show the measured farfield pattern of the optical system of MIAWARA-C at $22.235 \mathrm{GHz}$. These measurements were performed using two methods. A description of the first method using a laboratory setup with a rectangular horn as transmitting antenna is given in de Wachter et al. (2009). The measurements of the beam patterns reveal that the antenna pattern is almost identical for the antenna pointing to the zenith and to the horizon. There is a small asymmetry in the direction $\phi$ orthogonal to the plane of the scanning direction $\theta$ indicated in Fig. 2, which is attributed to the off-axis geometry of the mirror and expected from physical optics simulations. The asymmetry is uncritical for the measurement since it lies below the $-20 \mathrm{~dB}$ level.

A description of the second method using the Sun as a radiation source is given in Sect. 4. The result of this measurement is an antenna pattern at the elevation angle of the sun, which is somewhere inbetween the zenith and the horizon. Comparing the two techniques the measurements reveal a high degree of agreement.

\subsection{Correlation receiver}

MIAWARA-C uses a correlation receiver based on a concept known from radio astronomy, compare (Predmore et al., 1985) and Tiuri (1964). This set-up allows to compare two input signals in real-time. The correlation receiver of MIAWARA-C, schematically shown in Fig. 2, consists of two identical heterodyne receivers connected by a hybrid coupler with two inputs for two RF signals. One of these signals is the radiation from the sky and the other is coming from and internal calibration load. The two RF signals are split equally between the two identical radiometer chains by the $90^{\circ}$ hybrid coupler. In the receivers the noise signals are amplified, band limited and down converted to the frequency range of the spectrometer.

At $22 \mathrm{GHz}$ and an elevation angle of $25^{\circ}$ the sky has a brightness temperature between 10 and $20 \mathrm{~K}$ and therefore the noise temperature of the internal calibration load should be as low as possible. For MIAWARA-C a commercial available active cold load, a so-called Colfet similar to the one described in Frater and Williams (1981), is used. Laboratory tests showed its noise temperature to be around $140 \mathrm{~K}$.

The digital Correlation and Spectrum Analysis backend (CoSpAn) was realized in an Acqiris AC 240 board having two input channels and a fast Field Programmable Gate Array (FPGA).

The original FFT firmware for this spectrometer allowed for the calculation of the total power spectrum from time domain signals in real-time for one of the input channels. 

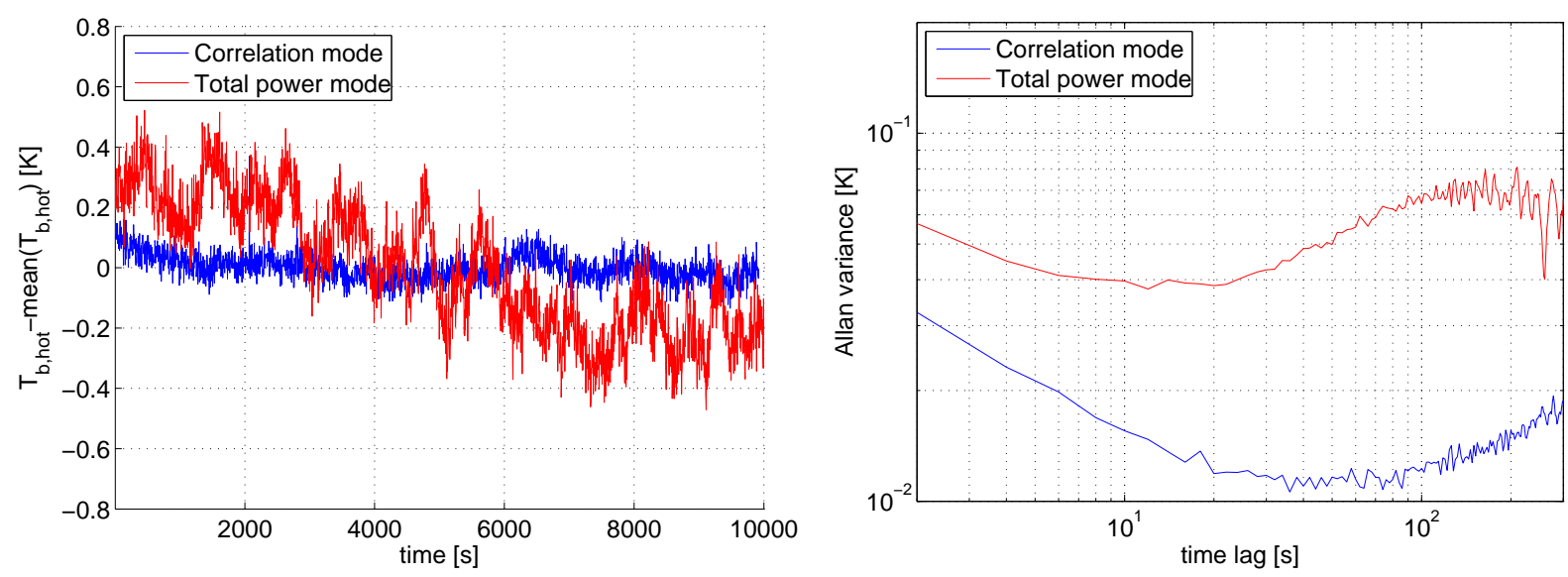

Fig. 5. Stability of the receiver of MIAWARA-C in the correlation- and the total power mode. Timeseries of the deviation of the calibrated data from the mean value (left) and Allan variance (right), $\tau=2 \mathrm{~s}$ and $\Delta \nu=400 \mathrm{MHz}$.

Descriptions of this concept can be found in Müller et al. (2006) and Benz et al. (2005).

For CoSpAn the FFT software has been customized to calculate a complex cross-correlation between the signals on the two input channels. In the FPGA core of the correlator each of the incoming signals is fast Fourier transformed separately and a complex multiplication is calculated. CoSpAn has a bandwidth of $500 \mathrm{MHz}$ and $2^{14}$ channels, which corresponds to a channel spacing of $30.5 \mathrm{kHz}$. The FWHM of each channel is also $30.5 \mathrm{kHz}$.

CoSpAn also allows for operation of the spectrometer in total power mode with the same bandwidth and resolution simultaneously for both inputs of the spectrometer.

A detailed mathematical description of the correlation receiver of MIAWARA-C can be found in Straub (2008). The output detected is proportional to the difference between the antenna temperature $T_{\mathrm{a}}$ and the brightness temperature of the Colfet $T_{\mathrm{b}, \text { Colfet }}$ :

$C(v)=a(v)\left(\frac{T_{\mathrm{b}, \operatorname{Colfet}}(v)-T_{\mathrm{a}}(v)}{2}\right)$

Here $a$ is a calibration constant proportional to the product of the gains of the two receiver chains, which makes the correlation receiver configuration less sensitive to gain drifts than the same receiver operated in total power mode. Figure 5 shows the result of stability tests using the MIAWARA$\mathrm{C}$ instrument with CoSpAn operated in cross-correlation and in total power mode, respectively. For these measurements the antenna was pointed to the hot load, described in Sect. 2.3, and data was sampled in $2 \mathrm{~s}$ intervals. The frequency mean over $400 \mathrm{MHz}$ bandwidth of the data was calibrated to brightness temperature of the absorber using a single measurement of a cold calibration target at the beginning of each test series. These measurements give an indication of the temperature fluctuations in degrees Kelvin, as displayed in the leftmost plot in Fig. 5. The Allan variance of the same dataset is shown in the plot on the right. Both representations of the stability of the receivers show that the correlation receiver is less affected by gain fluctuations than the total power receiver.

If the brightness temperature of the Colfet $T_{\mathrm{b} \text {,Colfet is }}$ known and constant the detected line signal can be calibrated with the following formula:

$T_{\mathrm{b}, \text { line }}=T_{\mathrm{b}, \text { Colfet }}-\frac{C_{\text {line }}}{C_{\text {hot }}}\left(T_{\mathrm{b}, \text { Colfet }}-T_{\text {hot }}\right)$

where $C_{\text {hot }}$ is the measurement of the brightness temperature of a calibration target at ambient temperature $T_{\text {hot }}$. This means the correlation receiver has the advantage that only one calibration measurement is necessary to determine the sky brightness temperature. How often the receiver needs to be calibrated depends on the stability of the system. In this instance the system stability is high and thus over $50 \%$ of the operating time can be devoted to actual measurement.

\subsection{Hot load}

The hot calibration target, the so-called hot load, of MIAWARA-C consists of a pyramid microwave absorber with a measured reflectivity below $-50 \mathrm{~dB}$. For the measurement of its physical temperature there are 16 temperature sensors of type LM92 placed in the pyramids of the absorber. The hot load itself is placed within the housing of MIAWARA-C and the antenna is directed towards it using a $45^{\circ}$ tilted mirror as indicated in Fig. 2. This set-up protects the hot-load from environmental influences like rain and sunshine.

\section{Balancing observation}

In Fig. 6 the plot on the left displays a spectrum calibrated with the method above. There are several artifacts visible. 

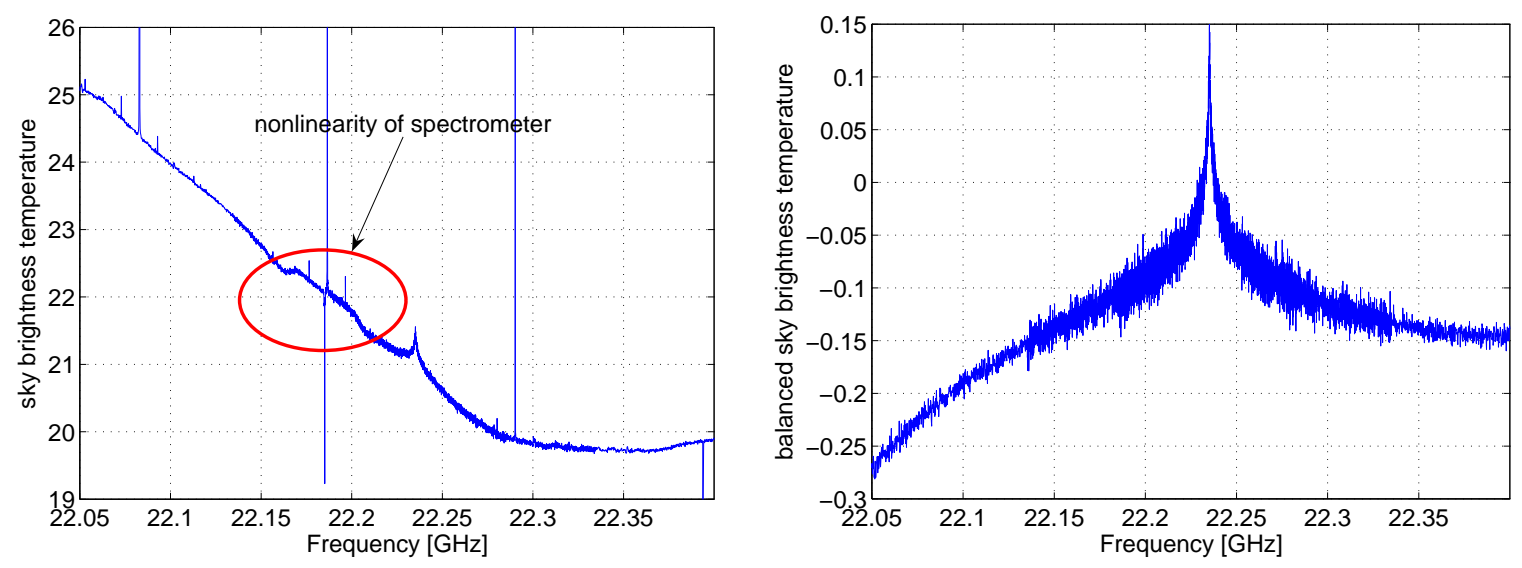

Fig. 6. Spectrum of MIAWARA-C after one day of integration calibrated with the method in Eq. (4) (left) and with the balancing calibration in Eq. (5) (right).

Firstly there are spikes affecting single channels that can easily be removed. Secondly there is a slope originating from standing waves within the system leading to a frequency dependence of $T_{\mathrm{b} \text {,Colfet which can likely be removed through }}$ fitting a superposition of sine waves to the spectrum. The third and most objectionable type of frequency dependent artifact are those that originate from power nonlinearities of the spectrometer. One such artifact is visible as a platforming at frequencies between 22.17 and $22.21 \mathrm{GHz}$ and is marked with a red circle. These platforming features are characteristic for the used FFT spectrometer as they are found in other radiometers using the same backend as well as in independent laboratory experiments. A sufficiently accurate method of controlling these spectrometer channel dependent non linearities could not be determined. For that reason additional external balancing, as described in Parrish et al. (1988), is needed to calibrate the output of MIAWARA-C.

The process of external balancing involves measuring a reference signal $C_{\text {ref }}$ that has the same intensity as the line measurement $C_{\text {line }}$, but which has little or no contribution from the water vapor line at $22 \mathrm{GHz}$. The difference between the two measurements is used to calculate the balanced sky brightness temperature $\Delta T_{\mathrm{b}}$. This is illustrated in the rightmost plot of Fig. 6 and is given by the following equation:

$\Delta T_{\mathrm{b}}=T_{\mathrm{b}, \text { line }}-T_{\mathrm{b}, \text { ref }}=\frac{C_{\text {line }}-C_{\text {ref }}}{C_{\text {hot }}-C_{\text {cold sky }}}\left(T_{\text {hot }}-T_{\mathrm{b}, \text { cold sky }}\right)(5)$

where $T_{\mathrm{b} \text {,line }}$ is the sky brightness temperature in the direction of the line measurement typically at $\sim 20^{\circ}$ elevation and $T_{\mathrm{b}, \text { ref }}$ is the temperature in the direction of the reference measurement. For the absolute calibration there are two targets; the hot load, and the sky under an elevation angle of $65^{\circ}$ which represents the cold load. The sky brightness temperature at a certain elevation angle can be approximated by using a simplified version of the RTE, Eq. (1), assuming the troposphere as a single layer with an effective temperature $T_{\text {eff }}$ :

$T_{\mathrm{b}}^{\text {sky }}(\theta)=T_{0} e^{-A_{\theta}^{\text {trop }} \tau_{\mathrm{z}}}+T_{\text {eff }}\left(1-e^{-A_{\theta}^{\text {trop }} \tau_{\mathrm{z}}}\right)$

where $\tau_{\mathrm{z}}$ is the opacity in zenith direction and $A_{\theta}$ is the airmass factor at the elevation angle $\theta$. This approach is used to find the brightness temperature of the sky used as the cold load. The determination of the tropospheric opacity is described in the next section. Trigonometrical considerations lead to the following formula for the airmass factors of the troposphere and middle atmosphere (will be used later):

$A^{\text {trop }}=\frac{\sqrt{(R+z)^{2}-R^{2} \cos ^{2} \theta}-R \sin \theta}{z}$

and

$A^{\mathrm{ma}}=\frac{\sqrt{(R+z+H)^{2}-R^{2} \cos ^{2} \theta}-\sqrt{(R+z)^{2}-R^{2} \cos ^{2} \theta}}{H}$

where $R$ is Earth's radius, $z$ is the height of the tropopause and $H$ is the height of the middle atmosphere from tropopause to mesopause.

The mean tropospheric temperature $T_{\text {eff }}$ can be estimated using the ambient temperature on the ground $T_{\mathrm{amb}}$ :

$T_{\mathrm{eff}}=c_{1}\left(T_{\mathrm{amb}}-273.15\right)+c_{0}$

where the values of the coefficients $c_{1}$ and $c_{0}$ are those derived experimentally by Han and Westwater (2000); namely $c_{1}=0.69$ and $c_{0}=266.3$.

The elevation angle for the line observation is chosen to achieve a maximum signal to noise ratio. The intensity of the observed water vapor signal depends on the path length through the middle atmosphere. This implies that the lower the elevation angle at which the line is observed, the longer the path length which leads to a stronger signal. Conversely, 
a lower elevation angle implies a stronger attenuation in the troposphere. Results from Haefele (2009) show that for tropospheric opacities below 0.2 the signal to noise ratio increases for decreasing elevation angles down to approximately $15^{\circ}$, beyond which it decreases further. Therefore for line observation elevation angles between $15^{\circ}$ and $25^{\circ}$ are required.

For the reference measurement the antenna of MIAWARA-C is directed to the zenith where the path length is a minimum. Microwave absorber is inserted into the beam to add signal which ensures that the measured intensity equals the emission of the sky at the elevation angle of the observation. During the balancing measurement the antenna beam is repeatedly switched between the fixed reference and adjusted line measurement in such a way that the elevation of the line measurement is manipulated in order to minimize $C_{\text {line }}-C_{\text {ref }}$.

The brightness temperature as measured in what is referred

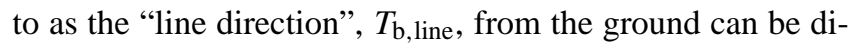
vided into the microwave background radiation $T_{0}$ plus the water vapor emission line originating in the middle atmosphere $T_{\mathrm{b}, \mathrm{z}}^{\mathrm{ma}}$, scaled with the airmass factor for the line measurement, and the continuum emission in the troposphere approximated as mean tropospheric temperature $T_{\text {eff }}$. For the part of the radiation originating above the tropopause, namely $T_{0}$ and $T_{\mathrm{b}, \mathrm{z}}^{\mathrm{ma}}$, one needs to consider the attenuation in the troposphere:

$T_{\mathrm{b}, \text { line }}=\left(T_{\mathrm{b}, \mathrm{z}}^{\mathrm{ma}} A_{\text {line }}^{\mathrm{ma}}+T_{0}\right) e^{-A_{\text {line }}^{\text {trop }} \tau_{\mathrm{z}}}+T_{\text {eff }}\left(1-e^{-A_{\text {line }}^{\text {trop }} \tau_{\mathrm{z}}}\right)$

Here $A$ is the airmass factor of the specified atmospheric layer $(\mathrm{ma}=$ middle atmosphere, trop $=$ troposphere $)$ for the specified measurement and $\tau_{\mathrm{z}}$ is the tropospheric opacity in the zenith direction.

The same separation between radiation originating above and below the tropopause can be made when regarding the sky brightness temperature in the reference direction. However, the influence of the microwave absorber in the beam needs to be taken into account:

$T_{\mathrm{b}, \mathrm{ref}}=T_{\mathrm{b}, \mathrm{ref}}^{*} t+(1-t) T_{\mathrm{abs}}$

with

$T_{\mathrm{b}, \text { ref }}^{*}=\left(T_{\mathrm{b}, \mathrm{z}}^{\mathrm{ma}} A_{\text {ref }}^{\mathrm{ma}}+T_{0}\right) e^{-A_{\text {ref }}^{\text {trop }} \tau_{\mathrm{z}}}+T_{\mathrm{eff}}\left(1-e^{-A_{\text {ref }}^{\text {trop }} \tau_{\mathrm{z}}}\right)$

where $T_{\mathrm{abs}}$ is the physical temperature of the reference absorber and $T_{\mathrm{b} \text {,ref }}^{*}$ the brightness temperature of the sky in the reference direction. The contribution of the absorber bar to the reference signal is regarded in terms of an equivalent transmission, $t$, of the reference absorber. This equivalent transmission of the reference bar indirectly depends on the opacity of the troposphere and is therefore not constant and needs to be determined for each measurement. The line signal from the middle atmosphere is present in the measurement in the reference direction. This is taken into account when the balanced spectrum is corrected for the attenuation in the troposphere, as will be discussed in Sect. 5.

\subsection{Tropospheric opacity}

To estimate the tropospheric opacity the iterative approach described in Han and Westwater (2000) is used. The basic premise of this method is to measure the sky at two or more different elevation angles and to relate the spectrometer outputs to sky brightness temperatures using the RTE (Eq. 1). For the tipping calibration of MIAWARA-C seven different angles in an elevation range between $25^{\circ}$ and $50^{\circ}$ are measured.

The opacity is then found by iteration of the following four steps:

1. The brightness temperature of the cold-sky calibration target $T_{\mathrm{b} \text {, cold sky }}$ is calculated using the RTE for the Troposphere (Eq. 6). For the first iteration an initial estimated value of 0.3 is used for the zenith opacity.

2. The measurements at the tipping elevations are calibrated using hot-cold calibration,

$$
T_{\mathrm{b}, \theta}=\frac{C_{\theta}-C_{\mathrm{hot}}}{C_{\mathrm{hot}}-C_{\text {cold sky }}}\left(T_{\mathrm{hot}}-T_{\mathrm{b}, \text { cold sky }}\right)+T_{\mathrm{hot}}
$$

where $T_{\mathrm{b}, \theta}$ is the microwave brightness temperature at the elevation angle $\theta, C_{\mathrm{x}}$ are the measured spectrometer outputs at the various targets and $T_{\text {hot }}$ is the physical temperature of the hot calibration target.

3. The opacity at the tipping angles is calculated from the calibrated measurements using the following relation:

$$
\tau_{\theta}=\ln \left(\frac{T_{\mathrm{eff}}-T_{0}}{T_{\mathrm{eff}}-T_{\mathrm{b}, \theta}}\right)
$$

4. The zenith opacity $\tau_{\mathrm{z}}$ is given by the slope of a linear fit of $\tau_{\theta}$ versus the airmass $A$, since in zenith direction $A=1$.

The linear fit must go through the origin of the coordinate system, since the opacity must be zero if there is no atmosphere. The iteration is stopped when the offset of the fit lies below $10^{-3}$. In practice the process presented here typically converges after the second iteration. The calibration of MIAWARA-C is divided into the two parts described above. Each cycle starts with a tipping curve to determine the tropospheric opacity and to have a measurement of the hot load and the cold sky calibration target. Following this the beam is continuously switched between the sky and the reference load for the balancing calibration. On average the calibration cycle takes $15 \mathrm{~min}$, of which approximately $38 \%$ is devoted to the line measurement.

\subsubsection{Validation of tipping-curve calibration}

To validate the opacities found with the tipping curve calibration a time series of tropospheric zenith opacity mean 

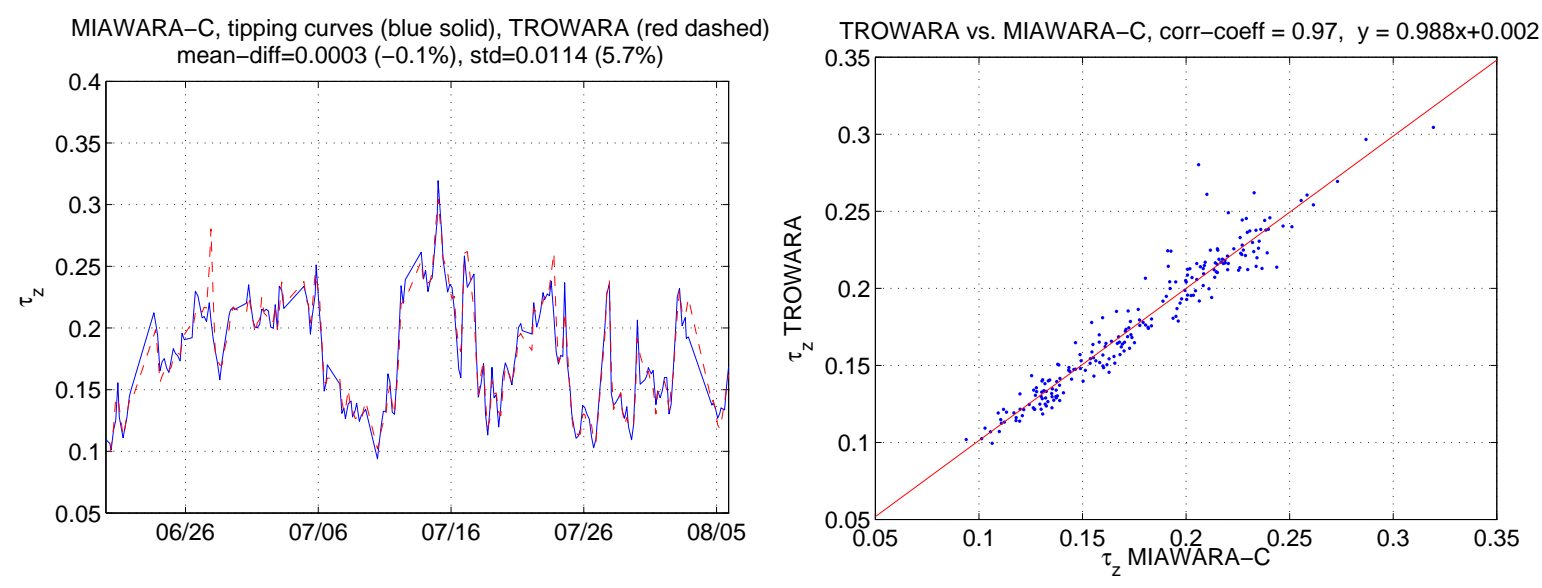

Fig. 7. Left panel: Time series of 4 hourly means of $\tau$ derived from MIAWARA-C tipping curves (mean value of $22.235 \pm 0.1 \mathrm{GHz}$ ) and TROWARA measurements at a constant elevation angle of $40^{\circ}$ (mean value of $22.235 \pm 0.2 \mathrm{GHz}$ ). Right panel: Correlation between the data obtained in the time period from 20 June to 5 August 2009.

values obtained during one and a half months in the Summer of 2009 in Bern are compared to those obtained using the TROWARA microwave radiometer which observed from the same location as MIAWARA-C but for a slightly different pointing (MIAWARA-C: south, TROWARA: south-east).

TROWARA monitors the sky brightness temperature at a constant elevation angle of $40^{\circ}$ in three frequency bands at 21.3, 22.2 and 31.5 GHz, (Mätzler and Morland, 2009). For this comparison only the channel at $22.2 \pm 0.2 \mathrm{GHz}$ is used.

The results of this comparison are displayed in Fig. 7 as a time series plot (left) and a scatter plot (right). TROWARA measures the sky brightness temperature at one second intervals while MIAWARA-C performs a tipping curve to determine the tropospheric opacity every $15 \mathrm{~min}$. Four hourly means of the two datasets are used to suppress the measurement noise and to reveal any possible systematic bias. The mean difference between the opacities determined with the two instruments is $0.1 \%$ with a standard deviation of $5.7 \%$, where the values obtained with MIAWARA-C are slightly higher than those from TROWARA. The correlation coefficient $r$ is 0.97 .

\subsubsection{Validation of the temperature of $\boldsymbol{T}_{\text {cold sky }}$}

The temperature of the cold sky determined by the tipping curve calibration is validated using liquid nitrogen calibration. For a comparison of the cold sky temperatures liquid nitrogen and tipping curve measurements are performed alternately. Three measurements, each lasting approximately three hours were performed on three different days during the Summer of 2009 in Bern. From these measurements the difference in the brightness temperature of the cold sky, $\Delta T_{\text {cold sky }}$, determined using the two different methods is found. The mean value of the difference is $0.7 \mathrm{~K}(1.8 \%$ of the mean temperature of the cold sky) with a standard deviation of $1.9 \mathrm{~K}$ (5.2\% of the mean temperature of the cold sky). It was found that due to reflexions on the air liquid nitrogen interface the microwave brightness temperature of liquid nitrogen was difficult to determine and thus it was concluded that the tipping curve calibration of $T_{\text {cold sky }}$ was of good quality. This result also shows that there is no obstacle in the beam at ambient temperature (e.g. the reference absorber) affecting measurement in cold sky direction.

\section{Validation of instrumental pointing}

An offset in the elevation pointing would lead to a systematic error in the calibration, namely when correcting the measured spectrum for use in the retrieval as described in Sect. 5 . For realistic values of the equivalent transmission of the reference absorber $t(0.85)$ and the tropospheric opacity $\tau(0.1)$ this is around 5\%/0.5\% of the tropospheric correction factor for a pointing offset of $1 \% .1^{\circ}$ in the angular range of our measurement, as Fig. 8 illustrates. To keep the error in the calibration below $1 \%$ the elevation pointing of MIAWARA$\mathrm{C}$ needs to be known with an accuracy of $0.2^{\circ}$. There are two different methods to determine the elevation pointing: the sky- and the Sun scanning. The sky scanning is a well known method to determine the pointing of microwave radiometers, compare Motte et al. (2008), while the more precise Sun scanning is adopted from radioastronomy. In the following section results from the Summer 2009 measurement campaign at Bern are presented. Spectral mean values are used for all calculations.

\subsection{Sky scanning}

For the sky scanning method the reference bar is removed in order to have an unobstructed view towards the zenith and approximately 19 measurements of the sky for 57 elevation 


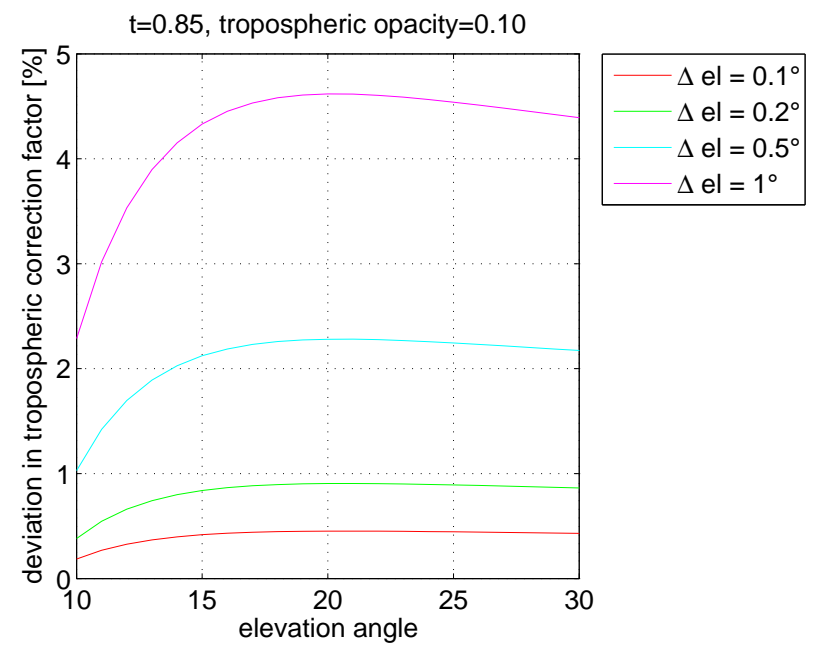

Fig. 8. Influence of a pointing offset in elevation on the tropospheric correction factor according to Eq. (15).

points between $30^{\circ}$ and $150^{\circ}$ and of the hot calibration target are performed. The sky at an elevation angle of $90^{\circ}$ is used as the cold calibration target. Its brightness temperature is determined using the RTE, where the opacity is found with a tipping curve calibration using the measurements at elevation angles between $30^{\circ}$ and $89^{\circ}$. The brightness temperature at the sky scanning angles $T_{\mathrm{b}, \theta}$ are calculated using hot-cold calibration. Calibrated sky scans performed during the Summer 2009 campaign are shown in Fig. 9 on top.

The zenith direction is where the temperature is the coldest. To find the minimum of the calibrated measurements the first derivative is determined, whereupon a third degree polynomial is fitted to the derivative and zeros are searched for. Figure 9 shows that the spread between the single scans is large, due to inhomogeneities in the tropospheric opacity. The fitting and the zero-search for the single scans results in an offset of $0.08^{\circ}$ with a standard deviation of $2.18^{\circ}$ while the same for the mean value, shown in the bottom panel of Fig. 9, results in a pointing offset of $0.01^{\circ}$ with an estimated standard deviation $2.18 / \sqrt{\# \text { scans }}=0.50^{\circ}$.

From this standard deviation it is concluded that this method is not suited to determine the elevation pointing with an accuracy of the required $0.2^{\circ}$.

\subsection{Sun scanning}

The ephemerides of the Sun are well known and therefore the Sun can be used to determine the elevation pointing of a radiometer by scanning a certain range (depending on the HPBW of the antenna) around the elevation of the Sun passing through the antenna beam.

Tropospheric properties must be taken into account when observing the Sun from the ground and therefore a tipping curve calibration is performed for every Sun scanning cycle from which the tropospheric opacity is determined. The ex-
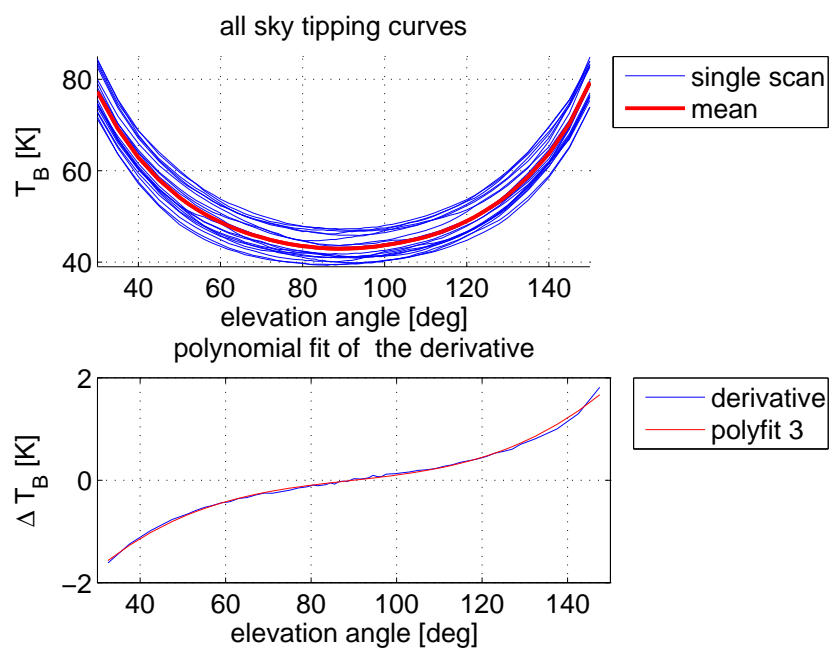

Fig. 9. Calibration of the elevation pointing of MIAWARA-C using the sky scanning method. Upper panel: Calibrated sky scans. Lower panel: First derivative of sky brightness temperature and polynomial fit of third degree.

pected sky brightness temperature at a certain elevation angle $T_{\mathrm{b}, \theta}^{\exp }$ is calculated using the RTE for the Troposphere. The actual sky brightness temperature $T_{\mathrm{b}, \theta}^{\text {act }}$ can be determined from a hot-cold calibration where the cold sky observation is performed at an elevation angle that is far away from the Sun (e.g. for a Sun elevation angle of $30^{\circ}$ an acceptable cold sky observation angle would be at approximately $60^{\circ}$ ). When the Sun passes the antenna beam, the actual sky brightness temperature deviates significantly from the expected one, since it is a linear combination of the brightness temperatures of the Sun and of the atmosphere.

As the Sun has an extent of approximately $0.5^{\circ}$ on the sky as observed from Earth the Sun scanning method may also be used to determine the shape of the antenna pattern (cf. Figs. 10 and 4).

To determine the pointing of the instrument the measurements are interpolated to a rectangular coordinate system, shown in Fig. 10 on the left, and a weighted mean value of the antenna pattern is taken in elevation direction. A Gaussian curve is then fitted to the data, as shown in Fig. 10 on the right. The best fit in the elevation direction is equal to the difference in the actual elevation angle of the instrument and the elevation angle assumed by the instrument's operating software. For the Summer 2009 campaign the pointing offset determined with the Sun scanning method is $0.001^{\circ}$. A sequence of ten Sun scans performed during the Lapbiat campaign in winter 2010 showed a standard deviation of $0.02^{\circ}$. There are no significant systematic errors expected from this method since the ephemerides of the Sun are known with a high precision. It is concluded, that the $2-\sigma$ error of the elevation pointing offset determined with this method is $0.04^{\circ}$ and therefore the Sun scanning is well suited to determine the instrumental pointing. 

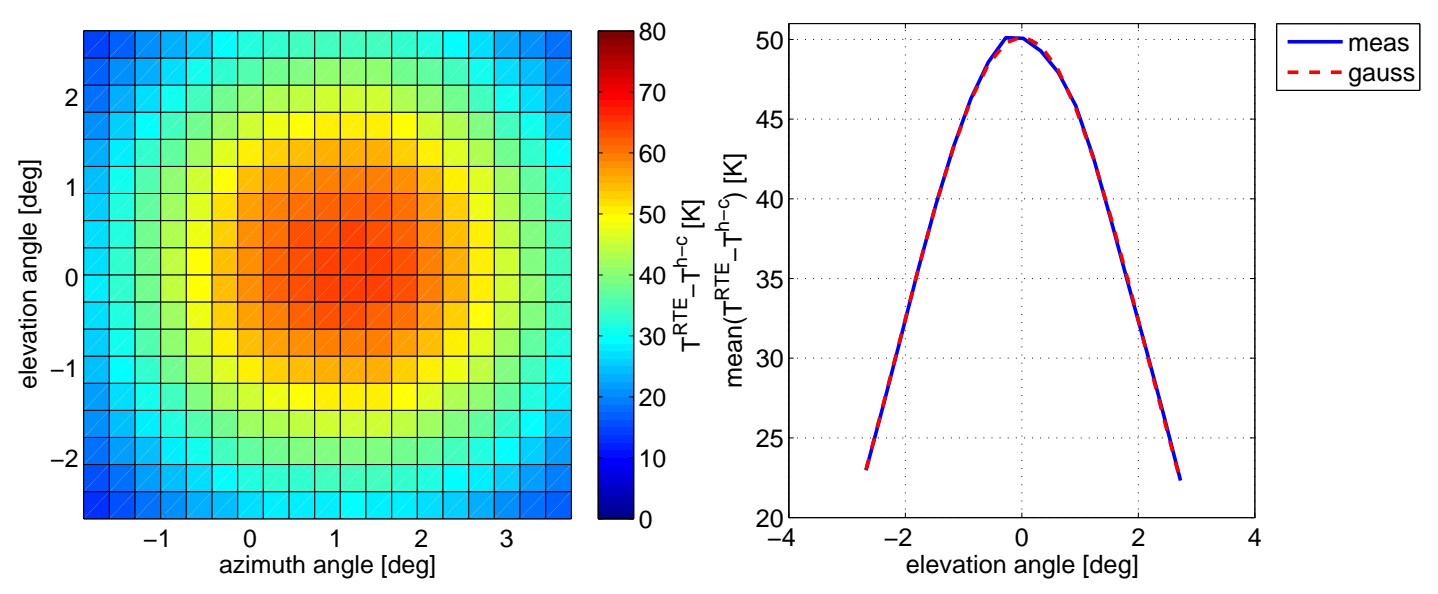

Fig. 10. Calibration of the elevation and azimuth pointing of MIAWARA-C using the Sun scanning method. Left panel: Result of Sun scanning interpolated to rectangular coordinate system relative to the Sun. Right panel: Gaussian curve fitted to weighted mean value of the antenna.

\section{First campaigns and results}

The first radiometric measurements from MIAWARA-C were performed at the end of 2008 in Bern, Switzerland $\left(47.0^{\circ} \mathrm{N}, 7.5^{\circ} \mathrm{E}, 550 \mathrm{~m}\right)$ after which it was shipped to the Zugspitze, Germany $\left(47.4^{\circ} \mathrm{N}, 11.0^{\circ} \mathrm{E}, 2650 \mathrm{~m}\right)$ in the beginning of January 2009 for the ARIS Campaign where it remained until the end of April of the same year. In Summer 2009 some minor alterations to the instrument housing were performed (the power supplies were transfered to a new compartment outside of the temperature stabilized part of the housing), upon which it was shipped to the Table Mountain Facility, USA $\left(34.4^{\circ} \mathrm{N}, 117.7^{\circ} \mathrm{W}, 2285 \mathrm{~m}\right)$ in the beginning of September for the MOHAVE 2009 campaign. From January to June 2010 MIAWARA-C was operated at the Finnish Meteorological Institute Arctic Research Centre in Sodankylä, Finland $\left(67.4^{\circ} \mathrm{N}, 26.6^{\circ} \mathrm{E}, 180 \mathrm{~m}\right)$ where it participated in the Lapbiat campaign. The Lapbiat campaign has to date been the longest and most successful measurement campaign in terms of the amount of data and therefore the data acquired during this time is used here for a first validation of MIAWARA-C against EOS/MLS.

For use in the retrieval we calculated the spectrum of emission in the zenith direction from the tropopause upward. The reason to use zenith direction for the retrieval is that for the line observation the elevation angle is not constant but is continuously adjusted to balance the reference load as the opacity of the troposphere is constantly changing. The tropopause level is chosen so that the information from the troposphere will be suppressed by the balancing technique. In order to determine such a spectrum that is independent of the tropospheric conditions $\Delta T_{\mathrm{b}, \mathrm{Z}}^{*}$ each balanced spectrum $\Delta T_{\mathrm{b}}$ is corrected for the tropospheric attenuation:

$\Delta T_{\mathrm{b}, \mathrm{z}}^{*}=\frac{\Delta T_{\mathrm{b}}}{A_{\mathrm{line}}^{\mathrm{ma}} e^{-A_{\text {line }}^{\text {trop }} \tau_{\mathrm{z}}}-t A_{\mathrm{ref}}^{\mathrm{ma}} e^{-A_{\mathrm{ref}}^{\text {trop }} \tau_{\mathrm{z}}}}-T_{\mathrm{b}, \text { res }}$ where according to Eq. (5) $\Delta T_{\mathrm{b}}=T_{\mathrm{b}, \text { line }}-T_{\mathrm{b} \text {, ref }}$ results by taking the difference of (Eqs. 10 and 11), taking into account that the spectra are balanced. This correction also takes into account that the line signal from the middle atmosphere is not only present in the line - but also in the reference measurement.

The equivalent transmission of the reference absorber $t$ is determined by solving Eq. (11):

$t=\frac{T_{\mathrm{b}, \mathrm{ref}}-T_{\mathrm{abs}}}{T_{\mathrm{b}, \mathrm{ref}}^{*}-T_{\mathrm{abs}}}$

where the total brightness temperature $T_{\mathrm{b} \text {,ref }}$ is found using hot-cold calibration from Eq. (13) and the sky brightness temperature in the reference direction $T_{\mathrm{b} \text {,ref }}^{*}$ using the RTE for the troposphere from Eq. (6).

$T_{\mathrm{b} \text {,res }}$ is a frequency dependent residuum term originating from the difference in the tropospheric emission in the line and the reference signal. The instrumental artifacts, known as the baseline, that originate from either the standing wave effects inherent to the receiver system or the frequency dependencies of the antenna pattern are also included in this term, which is dealt with during the retrieval process.

In order to obtain a signal-to-noise ratio from which a sufficiently accurate water vapor profile can be retrieved from the spectrum measured by MIAWARA-C, integrations of measurement times spanning from several hours up to days must be performed. The blue plot displayed in the top panel of Fig. 11 shows a typical one day balanced spectrum after the tropospheric correction has been applied. The difference in the noise level in the center and on the wings of the spectrum results from different binning, where there is no binning at the center and a binning of ten channels on the wings. For the profile retrieval the ARTS and QPack software packages, described in (Buehler et al., 2005) and (Eriksson et al., 2005), are used. ARTS is a modular program simulating 

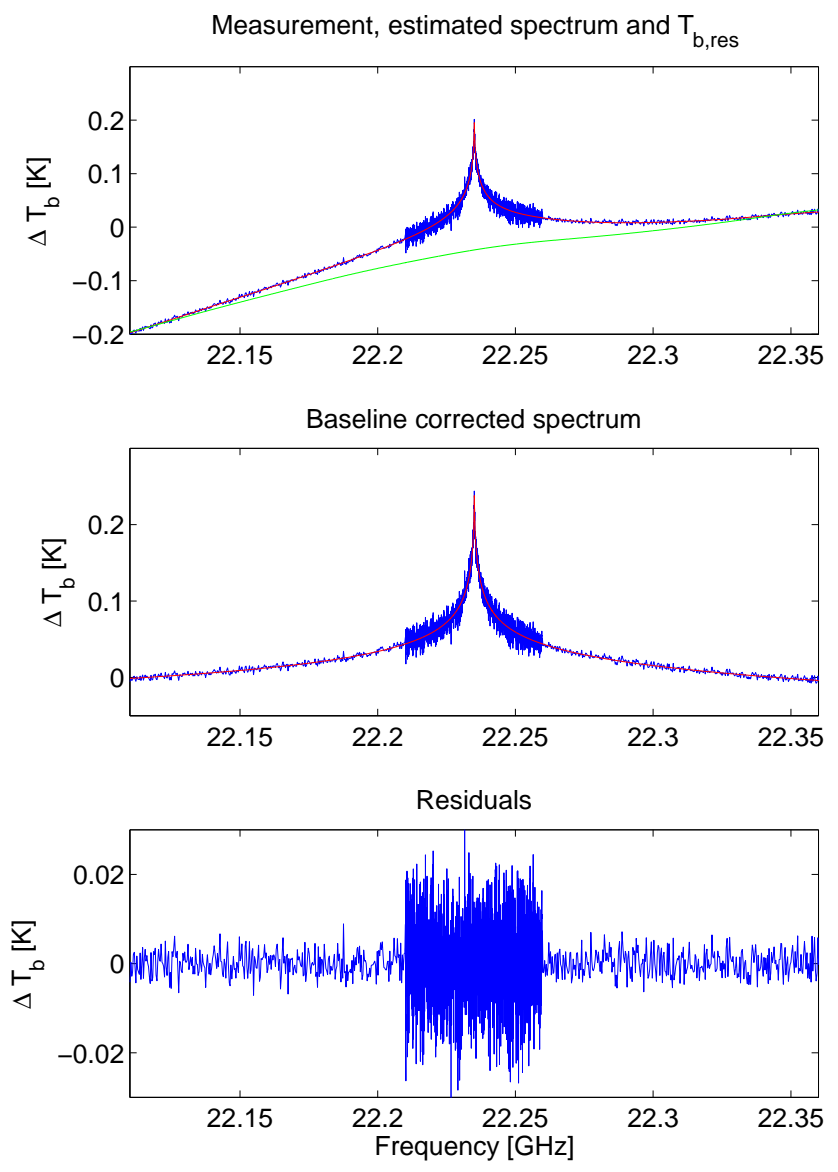

Fig. 11. Top panel: measured and estimated spectrum together with fit of $T_{b}$,res, middle panel: measured and estimated spectrum with fit of $T_{b, r e s}$ subtracted, bottom panel: residuals between estimation and measurement. The difference in the noise levels in different frequency ranges is due to a frequency binning of 10 channels used on the wings of the spectrum for data reduction.

atmospheric radiative transfer that can be used as a forward operator for the inverse model package Qpack. Qpack uses the optimal estimation model, described in (Rodgers, 2000), for the retrieval process. In the optimal estimation process the residuum term $T_{\mathrm{b} \text {,res }}$ is regarded as the second fitting parameter besides the water vapor distribution calculated with the forward model. $T_{\mathrm{b} \text {,res }}$ is estimated using a combination of polynomial and sinusoidal functions. The top panel of Fig. 11 shows the estimate spectrum in red and the fit of the residuum term in green. The middle panel shows the measured and the estimated spectrum with the fit of the residuum term subtracted and the bottom panel shows the residuals between measured and estimated spectrum.

Figure 12 on the left shows the profile retrieved from a spectrum with $250 \mathrm{MHz}$ bandwidth around the line center of the one day spectrum shown in Fig. 11. The error bars plotted give an upper limit for the sum of the systematic and the random error. The date of the presented profile was chosen
Table 2. Estimates of the errors of the relevant forward model parameters

\begin{tabular}{ll}
\hline Parameter & Estimated error \\
\hline Measurement noise & depends on integration time here $0.011 \mathrm{~K}$ \\
Temperature profile & $5 \mathrm{~K}$ \\
Calibration & $7 \%$ of factor for the tropospheric correction \\
Line intensity S & $8.7 \times 10^{-22} \mathrm{~m}^{2} \mathrm{~Hz}$ \\
Air broadening $\gamma_{\text {air }}$ & $1014 \mathrm{~Hz} / \mathrm{Pa}$ \\
\hline
\end{tabular}

near to a close overpass of the Microwave Limb Sounder MLS on the EOS Aura satellite (Lambert et al., 2007). For the retrieval of the MIAWARA-C $\mathrm{H}_{2} \mathrm{O}$ profiles shown here the temperature profile measured by MLS is used for the pressure-Temperature-altitude $(p T z)$ information, the line parameters are taken from JPL 1985 catalog (Poynter and Pickett, 1985) and the broadening parameters from Liebe (1989). The MLS climatology with a covariance linearly increasing from $10 \%$ at $0.681 \mathrm{hPa}$ to $55 \%$ at $0.005 \mathrm{hPa}$ is used as $\mathrm{H}_{2} \mathrm{O}$ apriori. The correlation of the apriori covariance is assumed to follow a Gaussian shape.

The plot in the center of Fig. 12 shows a comparison between the profile of MLS (red dash-dotted) and the one day profile of MIAWARA-C (blue solid). To achieve an identical vertical resolution between the two profiles the MLS profile was convolved with the averaging kernels and the apriori profile of MIAWARA-C (red solid). From these results it is concluded that the two profiles exhibit overall good agreement. The averaging kernels (AVK) plotted on the right in Fig. 12 show that the single day retrieval of MIAWARA-C can be trusted for altitudes between approximately 6 and $0.05 \mathrm{hPa}$, where the upper limit can be shifted higher by decreasing the measurement noise with longer integration times. The black dashed line shows the full width at half maximum (FWHM) of the AVK giving an indication of the altitude resolution of the retrieved profile.

The Qpack software package provides a tool for the error analysis of the retrieved data based on the calculation of perturbations for different forward model parameters. An estimation of the uncertainty of the relevant forward model parameters of MIAWARA-C is given in Table 2. These estimated values are based on those determined for MIAWARA given in Haefele (2009). It is desirable to provide an upper limit to the errors and thus a conservative calibration error value of $7 \%$ of the tropospheric correction factor is chosen. In Fig. 13 a plot of the vertically resolved estimated 2- $\sigma$ errors of the retrieved profiles is shown. The errors result from different families of uncertainty, namely measurement noise, calibration including pointing, spectroscopy, the temperature profile and the smoothing of the retrieval. The smoothing error can be ignored in the instance where the "true" state of the atmosphere is convolved with the AVK when it is compared to the retrieved profile of MIAWARA-C. The differentiation 

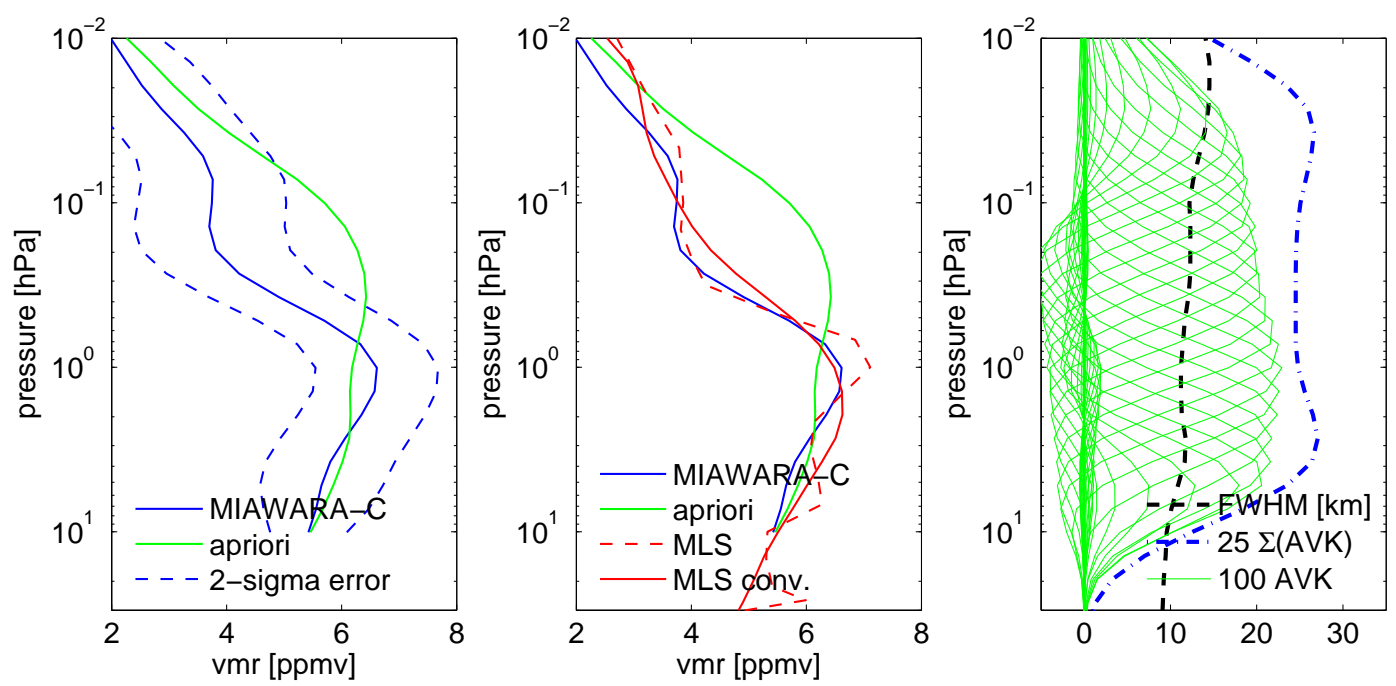

Fig. 12. Water vapor profile retrieved from the one day spectrum shown in Fig. 11 plotted together with the apriori profile and the upper limit for the systematic plus random 2- $\sigma$ error and compared to the profile of a close MLS overpass $\left(67.5^{\circ} \mathrm{N}, 21.0^{\circ} \mathrm{E}\right)$ in the middle plot. The averaging kernels of MIAWARA-C are shown on the right together with the FWHM and the sum of each of the kernels.

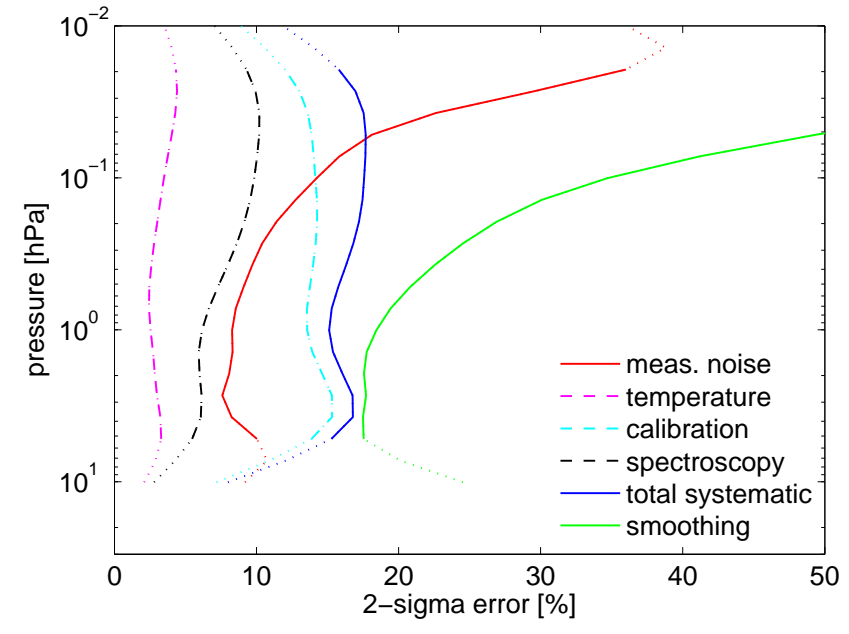

Fig. 13. Estimated 2- $\sigma$ errors in the retrieved profile resulting from different families of uncertainty.

between random and systematic error is challenging. Here all of the error sources except for the measurement noise and the smoothing error are considered as systematic even if the calibration and temperature errors are random to some extent. Therefore an approximate $16 \%$ systematic error for all altitudes is considered the upper limit while the measurement noise marks the lower limit for the random error.

The plots in Figs. 14 and 15 display comparisons between $\mathrm{H}_{2} \mathrm{O}$ profiles of MLS and 1-day MIAWARA-C retrievals acquired during the Lapbiat 2010 Campaign. The comparison period is from 15 January to 22 April. The criterion for a collocation of a MLS profile with the measurement site is $\pm 1^{\circ}( \pm 110 \mathrm{~km})$ in latitude and $\pm 5^{\circ}( \pm 460 \mathrm{~km})$ in longitude and results in 83 collocations for the 98 day comparison period. For both comparisons presented the profiles of MLS have been interpolated to the pressure grid of MIAWARA-C.

A time series of $\mathrm{H}_{2} \mathrm{O}$ volume mixing ratio as observed by the two instruments at six pressure ranges between 10 and $0.03 \mathrm{hPa}$ is shown in Fig. 14 and the relative difference between each profile of MIAWARA-C and the MLS profile closest in time is presented in Fig. 15. The agreement between the measurements of MLS and MIAWARA-C is within $5 \%$ for pressure altitudes between 6 and $0.05 \mathrm{hPa}$. The time evolution in $\mathrm{H}_{2} \mathrm{O}$ is similar for the measurements of both instruments at all altitudes.

\section{Conclusions}

MIAWARA-C is a new ground based radiometer for middle atmospheric water vapor, that combines a very compact optical system with a new type of correlation receiver using a digital high resolution spectrometer. The compact design and easy handling of the instrument make it well suited for use in measurement campaigns, as has already been shown for the ARIS, MOHAVE 2009 and Lapbiat campaigns.

Tipping curve measurements are used to determine the tropospheric opacity which is used to calculate the temperature of the cold calibration target, namely the sky under an elevation angle of $65^{\circ}$, and to correct the stratospheric signal for attenuation in the troposphere. The tropospheric opacities determined with MIAWARA-C are within $1 \%$ of those found with the tropospheric microwave radiometer TROWARA. Liquid nitrogen calibration was used to validate the cold-sky temperature and results showed it to be accurate to within $1 \mathrm{~K}$. This validation was repeated in the beginning of each 


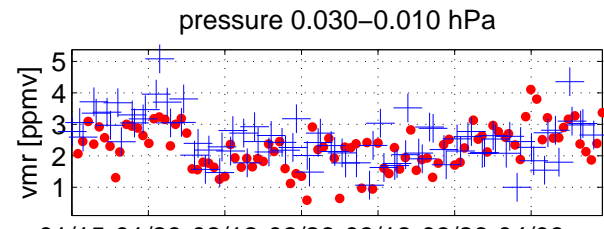

$01 / 1501 / 2902 / 1202 / 2603 / 1203 / 2604 / 09$

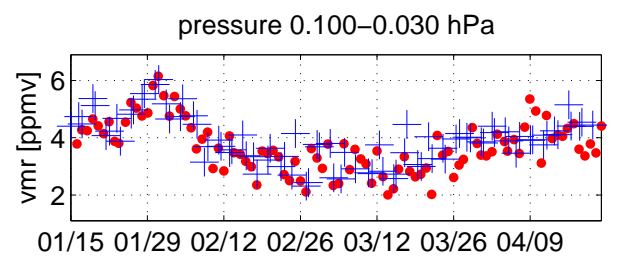

pressure $0.300-0.100 \mathrm{hPa}$

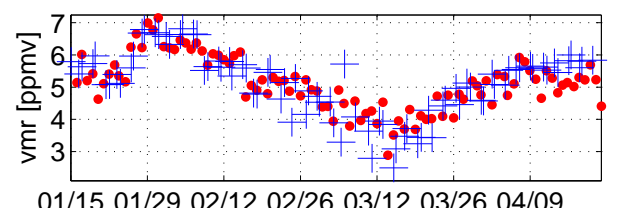

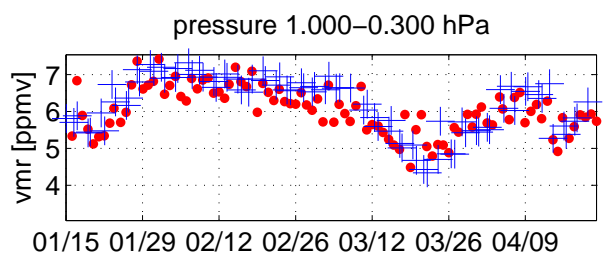
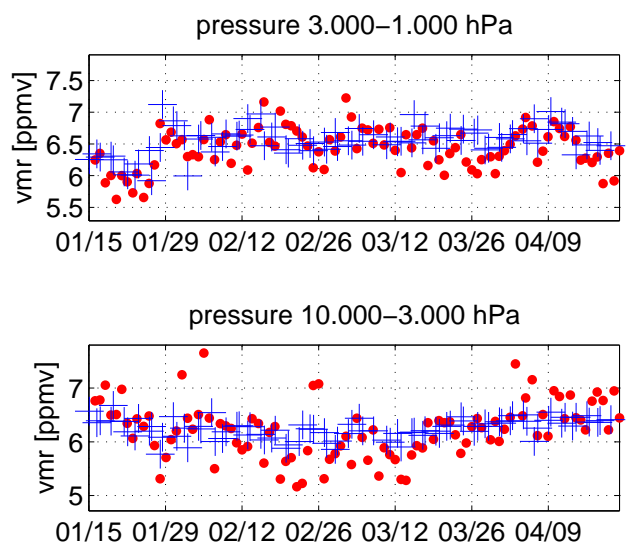

- MIAWARA-C + MLS

Fig. 14. Timeseries of middle atmospheric $\mathrm{H}_{2} \mathrm{O}$ for 6 pressure ranges between 10 and $0.01 \mathrm{hPa}$ as observed by MIAWARA-C and MLS during the Lapbiat campaign. A mean value of the $\mathrm{H}_{2} \mathrm{O}$-vmr within the pressure ranges indicated is used to account for the different altitude resolutions of the two instruments.
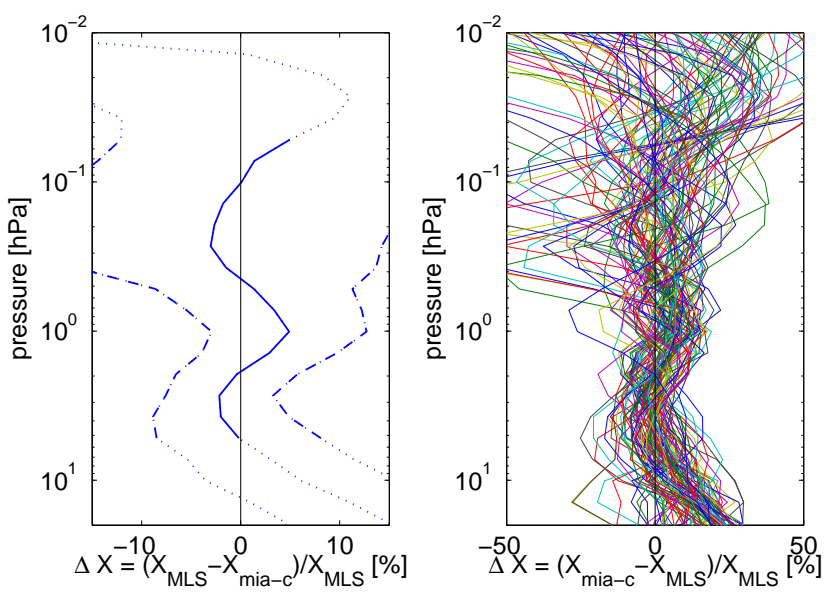

Fig. 15. Relative difference between the measurements of MIAWARA-C and MLS, mean value and standard deviation (left) and single profile comparisons (right).

campaign and every time the reference absorber is changed to assure there is no contamination from the reference bar in the antenna beam while measuring the cold sky calibration target.

For the validation of the elevation pointing a Sun and the sky scanning method are presented. Considerations of the standard deviations of the two methods revealed that the Sun scanning is a extremely accurate method which allowes to determine the instrumental pointing to the level of a 2- $\sigma$ ran- dom error of $0.04^{\circ}$ while the precision of the sky scanning method is not sufficient to determine the pointing offset to an accuracy of the required $0.2^{\circ}$.

The example of a one day spectrum and retrieval during the Lapbiat campaign show that MIAWARA-C measures spectra with no severe baseline artifacts. A first validation comparing 98 days of MIAWARA-C data to the profiles of MLS indicates that the mean difference between the profiles is below $5 \%$ at altitudes between 6 and $0.05 \mathrm{hPa}$, which is the pressure range for the retrieved profiles from MIAWARA-C that contains the greatest amount of information on the measured spectra. A comparison between time series at different pressure ranges shows that MIAWARA-C and MLS measure similar time evolutions of water vapor volume mixing ratio. The evaluation of the data acquired during the ARIS and the MOHAVE 2009 campaigns will allow for future comparisons of MIAWARA-C profiles with several other instruments.

Acknowledgements. This work has been supported by the Swiss National Science Foundation grant number 200020-124387. Participation at the Lapbiat campaign was funded through the EU Sixth Framework Programme, Lapland Atmosphere-Biosphere Facility (LAPBIAT2). We thank the team of the Finnish Weather Service for their hospitality and support during the campaign. Particularly we like to thank the Bern University Research Foundation for funding the weather station of MIAWARA-C.

Edited by: J. Notholt 


\section{References}

Benz, A. O., Grigis, P. C., Hungerbühler, V., Meyer, H., Monstein, C., Stuber, B., and Zardet, D.: A broadband FFT spectrometer for radio and millimeter astronomy, Astron. Astrophys., 442, 767773, 2005.

Buehler, S. A., Eriksson, P., Kuhn, T., von Engeln, A., and Verdes, C.: ARTS, the atmospheric radiative transfer simulator, J. Quant. Spectrosc. Ra., 91, 65-93, 2005.

de Wachter, E., Murk, A., Straub, C., Haefele, A., Ka, S., Oh, J. J., and Kämpfer, N.: Effects of Resonances in Corrugated Horn Antennas for a $22 \mathrm{GHz}$ Balancing Radiometer, IEEE Geoscience and Remote Sensing Letter, 6, 3-7, 2009.

de Wachter, E., Haefele, A., Kämpfer, N., Ka, S., Lee, J. E., and Oh, J. J.: The Seoul Vapour Radiometer for the Middle Atmosphere; Calibration, Retrieval and Validation, Transactions on Geoscience and Remote Sensing, doi:10.1109/TGRS.2010.2072932, 2010.

Deuber, B., Kämpfer, N., and Feist, D.: A New 22-GHz Radiometer for Middle Atmospheric Water Vapor Profile Measurements, IEEE Transactions on Geoscience and Remote Sensing, 42, 974984, 2004.

Eriksson, P., Jiménez, C., and Buehler, S. A.: Qpack, a general tool for instrument simulation and retrieval work, J. Quant. Spectrosc. Ra., 91, 47-64, 2005.

Forkman, P., Eriksson, P., and Winnberg, A.: The $22 \mathrm{GHz}$ radioaeronomy receiver at Onsala Space Observatory, J. Quant. Spectrosc. Ra., 77, 23-42, 2003.

Frater, R. H. and Williams, D. R.: An Active "Cold" Noise Source, IEEE Transactions on Microwave Theory and Techniques, 29, 344-347, 1981.

Haefele, A.: Measurements of Tropospheric, Stratospheric and Mesospheric Water Vapor by Ground Based Microwave SpectroRadiometry, Ph.D. thesis, University of Bern, 2009.

Han, Y. and Westwater, E. R.: Analysis and Improvement of Tipping Calibration for Ground-Based Microwave Radiometers, IEEE Transactions on Geoscience and Remote Sensing, 38, 1260-1276, 2000.

Lambert, A., Read, W. G., Livesey, N. J., Santee, M. L., Manney, G. L., Froidevaux, L., Wu, D. L., Schwartz, M. J., Pumphrey, H. C., Jimenez, C., Nedoluha, G. E., Cofield, R. E., Cuddy, D. T., Daffer, W. H., Drouin, B. J., Fuller, R. A., Jarnot, R. F., Knosp, B. W., Pickett, H. M., Perun, V. S., Snyder, W. V., Stek, P. C., Thurstans, R. P., Wagner, P. A., Waters, J. W., Jucks, K. W., Toon, G. C., Stachnik, R. A., Bernath, P. F., Boone, C. D., Walker, K. A., Urban, J., Murtagh, D., Elkins, J. W., and Atlas, E.: Validation of the Aura Microwave Limb Sounder middle atmosphere water vapor and nitrous oxide measurements, J. Geophys. Res., 112, D24S36, doi:10.1029/2007JD008724, 2007.

Liebe, H. J.: MPM - An atmospheric millimeter-wave propagation model, Int. J. Infrared. Milli., 10, 631-650, 1989.

Mätzler, C. and Morland, J.: Refined Physical Retrieval of Integrated Water Vapor and Cloud Liquid for Microwave Radiometer Data, IEEE Transactions on Geoscience and Remote Sensing, 47, 1585-1594, 2009.

Milz, M., von Clarmann, T., Fischer, H., Glatthor, N., Grabowski, U., Höpfner, M., Kellmann, S., Kiefer, M., Linden, A., Tsidu, G. M., Steck, T., Stiller, G. P., Funke, B., López-Puertas, M., and Koukouli, M. E.: Water vapor distributions measured with the Michelson Interferometer for Passive Atmospheric Sounding on board Envisat (MIPAS/Envisat), J. Geophys. Res., 110, D24307, doi:10.1029/2005JD005973, 2005.

Motte, E., Ricaud, P., Gabard, B., Niclas, M., and Gangneron, F.: A 22-GHz Mobile Microwave Radiometer (MobRa) for the Study of Middle Atmospheric Water Vapor, IEEE Transactions on Geoscience and Remote Sensing, 46, 3104-3114, 2008.

Müller, S., Murk, A., Monstein, C., Kämpfer, N., and Meyer, H.: Microwave remote sensing of stratospheric trace gases using digital Fast Fourier Transform spectrometers, in: GeMiC $2006-$ Conference Proceedings, 2006.

Murtagh, D., Frisk, U., Merino, F., Ridal, M., Jonsson, A., Stegman, J., Witt, G., Eriksson, P., Jiménez, C., Megie, G., de la Noë, J., Ricaud, P., Baron, P., Pardo, J. R., Hauchcorne, A., Llewellyn, E. J., Degenstein, D. A., Gattinger, R. L., Lloyd, N. D., Evans, W. F., McDade, I. C., Haley, C. S., Sioris, C., von Savigny, C., Solheim, B. H., McConnell, J. C., Strong, K., Richardson, E. H., Leppelmeier, G. W., Kyrölä, E., Auvinen, H., and Oikarinen, L.: An overview of the Odin atmospheric mission, Can. J. Phys., 80, 309-319, 2002.

Nedoluha, G. E., Bevilacqua, R. M., Gomez, R. M., Thacker, D. L., Waltman, W. B., and Pauls, T. A.: Ground-based measurements of water vapor in the middle atmosphe, J. Geophys. Res., 100, 2927-2937, 1995.

Nedoluha, G. E., Gomez, R. M., Hicks, B. C., Bevilacqua, R. M., Russell, J., Connor, B. J., and Lambert, A.: A comparison of middle atmospheric water vapor as measured by WVMS, EOS-MLS, and HALOE, J. Geophys. Res., 112, D24S39, doi:10.1029/2007JD008757, 2007.

Parrish, A., Dezafra, R. L., Solomon, P. M., and Barrett, J. W.: A ground-based technique for millimeter wave spectroscopic observations of stratospheric trace constituents, Radio Sci., 23, 106-118, 1988.

Plumb, R. A.: A “tropical pipe" model of stratospheric transport, J. Geophys. Res., 101, 3957-3972, 1996.

Poynter, R. L. and Pickett, M.: Submillimeter, millimeter, and microwave spectral line catalog, Appl. Opt., 24, 2235-2240, 1985.

Predmore, C. R., Erickson, N. R., Huguenin, G. R., and Goldsmith, P. F.: A Continuous Comparison Radiometer at $97 \mathrm{GHz}$, IEEE Transactions on Microwave Theory and Techniques, 33, 44-51, 1985.

Rodgers, C. D.: Inverse Methodes for Atmospheric Soundings, World Scientific Publishing Co. Pte. Ltd, Singapore, 2000.

Straub, C.: Development of a $22 \mathrm{GHz}$ correlating Radiometer for the observation of Stratospheric Water Vapor, Master's thesis, University of Bern, 2008.

Straub, C., Murk, A., Teniente, J., and Kämpfer, N.: Optical design for a compact $22 \mathrm{GHz}$ radiometer for middle atmospheric water vapor, in: EUCAP 2007 Conference proceedings, 2007.

Teniente, J., Gonzalo, R., and del Rio, C.: Choked Gaussian Antenna: Extremely Low Sidelobe Compact Antenna Design, IEEE Antennas and Wireless Propagation Letters, 1, 200-202, 2002.

Thacker, D. L., Bevilacqua, R. M., Waltman, W. B., Pauls, T. A., Gomez, R. M., Nedoluha, G. E., and Schwartz, R. R.: Groundbased sensing of water vapor in the stratosphere and mesosphere, IEEE Transactions on Instruamentation and Measurement, 44, 355-359, 1995.

Tiuri, M. E.: RadioAstronomyReceivers, IEEE Transactions on Antennas and Propagation, 12, 930-938, 1964.

Waters, J., Froidevaux, L., Harwood, R., Jarno, R., Pickett, H., 
Read, W., Siegel, P., Cofield, R., Filipiak, M., Flower, D., Holden, J., Lau, G., Livesey, N., Manney, G., Pumphrey, H., Santee, M., Wu, D., Cuddy, D., Lay, R., Loo, M., Perun, V., Schwartz, M., Stek, P., Thurstans, R., Boyles, M., Chandra, S., Chavez, M., Chen, G.-S., Chudasama, B., Dodge, R., Fuller, R., Girard, M., Jiang, J., Jiang, Y., Knosp, B., LaBelle, R., Lam, J.,
Lee, K., Miller, D., Oswald, J., Patel, N., Pukala, D., Quintero, O., Scaff, D., Snyder, W., Tope, M., Wagner, P., and Walch, M.: The Earth Observing System Microwave Limb Sounder (EOS MLS) on the Aura satellite, IEEE Transactions on Geoscience and Remote Sensing, 44, 1075-1092, 2006. 